\title{
Treatment of Posterior Malleolus Fracture through Posterolateral Approach
}

\author{
Sah Ganesh Kumar, Jun Lu*, Singh Ratish, Sinkemani Arjun, Karki Sundar, Jiming Liang \\ Department of Orthopedic Surgery, Zhongda Hospital Affiliated to Southeast University, Nanjing, China \\ Email: *feonix1976@126.com
}

How to cite this paper: Kumar, S.G., Lu, J., Ratish, S., Arjun, S., Sundar, K. and Liang, J.M. (2019) Treatment of Posterior Malleolus Fracture through Posterolateral Approach. Open Journal of Orthopedics, 9 , 67-80.

https://doi.org/10.4236/ojo.2019.94007

Received: February 28, 2019

Accepted: March 25, 2019

Published: March 28, 2019

Copyright $\odot 2019$ by author(s) and Scientific Research Publishing Inc. This work is licensed under the Creative Commons Attribution International License (CC BY 4.0).

http://creativecommons.org/licenses/by/4.0/

\begin{abstract}
Posterior malleolus fractures are quite common and usually result from rotational ankle injuries. For the management of posterior malleolus fractures, more studies are still required, though it is already well-recognized for medial and lateral structure. Therefore, fracture of posterior malleolus is striking subject of study among orthopedic surgeons. Most orthopedic surgeons recommend fixing the posterior malleolus fracture if it is larger than $25 \%$ to $33 \%$ of the distal articular surface. Further attention is required for the reduction and fixation of fractures involving posterior malleolus. Several approaches and methods for fixation of posterior malleolus have been defined in the literature. Previously, the most common method of fixation of the posterior malleolus is by indirect reduction and anteroposterior screws, it is minimally invasive, the anterior incision does not allow satisfactory visualization of the fragment, so good anatomical reduction is difficult to achieve thorough this approach. Operative management goals to reach a stable ankle with maximal function, decrease the risk of post-traumatic degenerative changes, and diminish the risk of complication. Nowadays, posterolateral approach is gaining the popularity due to adequate visualization and accurate anatomic reduction.
\end{abstract}

\section{Keywords}

Ankle Fracture, Posterior Malleolus Fracture, Trimalleolus Fracture,

Posterolateral Approach

\section{Introduction}

Ankle fractures are injuries with an annual occurrence of approximately 100/ 100,000 person yearly [1] [2] [3]. Among which about $7 \%$ to $44 \%$ are accompanied by posterior malleolus fracture, which are rarely seen alone [4] [5]. Usually it results from rotation of ankle and accompanying typically with fractures of 
single or both malleoli. Typical rotational mechanism of the ankle fracture may lead to the rupture of the posterior inferior tibiofibular ligament (PITFL) or create an avulsion fracture of the posterior tibial margin. It is also defined as the posterior malleolus fracture or Volkmann's fracture. Radiological study of the posterior tibial rim fragment was initially studied by Chaput in 1907 [6] followed by Destot who further introduced the term "malle'ole poste'rieure" (posterior malleolus [PM]) in 1911. In 1915, Cotton described a new type of ankle fracture eventually named after him which was a bimalleolus fracture along with a fracture of PM [7]. However, in 1836 this type of fracture had been previously described by Adams [8]. Henderson introduced the term trimalleolus fracture in 1932 [9].

PM fractures are generally neglected due to the spontaneous reduction of these fragments after open reduction of the lateral malleolus through ligamentotaxis done by the posterior-inferior tibiofibular ligament (PITFL). Some studies have shown the importance of PM on the stability of tibiofibular syndesmosis (TFS) and the ankle joint [10] [11] [12]. Recent literature shows a changing tendency towards the anatomical correction of the joint, based on the presence of intra-articular step-off rather than the size of the posterior fragment [13] [14] [15].

Still, fixation of the posterior malleolus fractures remains an area of controversy in orthopedic surgery. No consensus has provided the best methodology for reduction and stabilizes the posterior tibial malleolus. The standard intervention for posterior malleolus fracture fixation involving $>25 \%-35 \%$ of the articular surface of distal tibia should be internally fixed [16] [17]. On the contrary, the need for fixation of fragments $<25 \%$ has not yet been clarified. Inadequate reduction of ankle fracture leads to early osteoarthritis, resulting in pain and loss of function of the ankle joint.

The recent advancement has evolved from simple closed reduction and casting, to open reduction and internal fixation. The literature describes various approaches for fixation of posterior malleolus. Traditionally, method of posterior malleolus fractures fixation is indirect reduction and an anteroposterior screw. Although minimally invasive, the anterior incision has limited visualization of the fragments further hindering a proper anatomic reduction leading to poor prognosis [18]. Therefore, posterolateral approach is gaining popularity due to adequate visualization and accurate anatomic reduction.

\section{Classification}

Decades of research into ankle fracture provides multiple classifications focusing on the mechanism of injury along with correlation with the pattern of the fractures. The most common classifications are those of Lauge-Hansen and Danis-Weber. The consideration of both classifications is desirable, as a surgeon should be able to establish a correlation between the fracture, mechanism of an injury and an optimal treatment method. 


\subsection{Lauge-Hansen Classification}

The most commonly accepted classification is the generic one made by LaugeHansen in 1984. It was based on experimental, clinical and radiological findings with the fracture type depending on parameters such as the position of the foot and the direction of the force at the time of injury. The term of Lauge-Hansen's classification is basically a cadaveric study [19] (Table 1 categories and stages of Lauge-Hansen classification system).

\subsection{Danis-Weber Classification}

On 1949 Robert Danish published "Théorie et pratique de l'ostéosynthèse" providing an early basis to classification. Further on 1972-Bernhard Georg Weber developed and popularized classification system still used in practice today. The Danis-Weber classification is essentially based on radiographic criteria.

The position of the distal fibular fracture in relation to the syndesmosis of the ankle joint were taken into consideration. After a detailed study three categories were created:

Type A fracture: below the level of the tibial plafond (syndesmosis) and may be associated with oblique or vertical medial malleolus fractures

Type B fractures: at the level of the tibial plafond (syndesmosis) and extend proximally in an oblique fashion

Type C fractures: proximal to the level of the tibial plafond and often have an associated syndesmotic injury. It can be associated with medial malleolus fractures or injury to the deltoid ligament [19] [20] [21].

\section{Mechanism of Injury}

The rotational injury is the most typical mechanism of leading to a fracture of the posterior malleolus. Lauge-Hansen classification system describes the progression of injury around the ankle joint in a staged sequence. The supination-external rotation injury pattern has an initial anterior inferior tibiofibular ligament injury (stage 1) followed by fibula (stage 2) which could lead to PITFL or fracture of the posterior malleolus (stage 3) and finally the medial ankle (stage 4). Posterior malleolus fracture also witnessed in the pronation-abduction. Here, the force of abduction creates avulsion of the syndesmosis with lateral malleolus failure which may also result in a fracture of the posterior malleolus as well. Although rare, there are few incidences of certain combined injury mechanism where an axial load and shear fracture off the posterior malleolus, which commonly occurs in falls from a height and road traffic accident. A torsional injury that results in a spiral distal tibia fracture also inflicts posterior malleolus fracture commonly seen with an associated high fibula fracture [22] [23].

\section{Diagnosis}

For the diagnosis of ankle fracture a proper history taking, thorough clinical examination and intense radiological study are needed. History of the incident 
Table 1. Lauge-Hansen classification [19].

\begin{tabular}{l}
\hline \\
1) Injury of the anterior inferior tibiofibular ligament (AITFL) \\
2) Oblique/spiral fracture of distal tibia \\
3) Injury of the posterior inferior tibiofibular ligament (PITFL) \\
4) Medial malleolus fracture or injury to the deltoid ligament \\
1) Transverse fracture of the distal fibula \\
2) Vertical fracture of medial malleolus \\
1) Medial malleolus fracture or injury to the deltoid ligament \\
2) Injury of the anterior inferior tibiofibular ligament (AITFL) \\
3) Oblique or spiral fracture of the fibula proximal to the tibial \\
plafond \\
4) Injury of the posterior inferior tibiofibular ligament (PITFL) or \\
avulsion of the posterior malleolus \\
1) Medial malleolus fracture or injury to the deltoid ligament \\
2) Injury of anterior inferior tibiofibular ligament \\
3) Transverse or comminuted fracture of the fibula proximal to the \\
tibial plafond
\end{tabular}

focuses on the details shedding a light on the mechanism of injury with the pre-existing medical status, and economic condition which are of great indicators for decision-making and planning the mode of treatment. The physical examination helps to recognize the emergency condition, open fracture, compromised neurovascular status and compartment syndrome. Local tenderness and ankle stability should not be ignored. Radiology is essential in defining involved injured structures which is important for the plan of treatment which is a supporting parameter for a better quality of the reduction.

The initial radiograph includes at least three projections: AP (anterior-posterior), lateral and mortise view. Computed tomography (CT) is helpful to determine fragments with the location of the major fragment. Haraguchi et al. proposed a calculation method for fragment size based on CT images which are considered more accurate [24]. For the ligament and tendon injury MRI proves to be more helpful.

\section{Treatment}

The treatment option consists of conservative or operative treatment which depends on the fracture type, state of the circulation and skin condition, general condition of the patient with numerous possible complications. Previously, fractures of the posterior malleolus fractures were treated nonoperatively. There are few studies suggesting closed reduction with cast immobilization should be reserved for non-displaced, stable fractures, anatomically reduced fractures and for patients with the poor medical condition.

From 1950s onward, surgical recommendation of the posterior malleolus fracture has varied. Still, the proposed guidelines have certain drawbacks such as lack of poor-quality studies, inaccurate evaluation of fragment size, added not all surgical cases are fixed anatomically. Based on previous studies it has been recommended to surgically fix posterior malleolus fractures that are $>25 \%-35 \%$ of 
the articular surface of the tibia [25].

Previously, ankle instability was the first indication for internal fixation of the PM [26] [27] [28]. Eventually, attention was paid to the importance of joint congruity and restoration of the articular surface of the tibial pilon [29] [30]. Recently, emphasis has been put on stability of the tibiofibular mortise and reduction of the distal fibula into the fibular notch [31]. A longer period followed a decisive factor for indication to surgery as the size of the articular surface of the fragment and its displacement [32] [33].

Although indications for fixation of the posterior malleolus still remains controversial, the existing indications are evolving which includes fractures involving $>25 \%$ to $33 \%$ of the articular surface, displacement $>2 \mathrm{~mm}$, ankle instability with concomitant syndesmotic injury, and persistent posterior subluxation of the talus.

\section{Operative Treatment: Posterolateral Approach}

\section{Surgical Technique}

The preferred surgical process is the prone or semi-prone position although patients could lie on their side, if there is no medial malleolus fracture. Under anesthesia, $8-12 \mathrm{~cm}$ longitudinal incision is made between the lateral border of Achilles tendon and the posterior margin of the fibula. Therefore, the surgeon must be careful to avoid injuring the sural nerve. A sharp incision is made through subcutaneous tissue to visualize the nerve at the lateral border of the Achilles tendon, which is identified as approximately $10 \mathrm{~cm}$ proximal to the tendon insertion. Deep dissection was continued between the flexor hallucis longus tendon medially and both long and short peroneal tendons laterally to expose the posterior malleolus fragments.

Hereafter, the posterolateral fragment is gently mobilized with careful attention for keeping the posterior tibiofibular ligament intact. After fracture surfaces debris is cleared, the fracture is reduced and temporarily fixed by K-wires posteriorly to anteriorly, abutting the articular surface. Care should be taken to clean and define the fracture edge, and not to devascularize the fragment. Intercalary fragments of sufficient size and cartilage quality are reduced towards the tibial plafond using the talus as a model and fixed with $\mathrm{K}$-wires or resorbable pins. Comminuted osteochondral fragments not amenable to fixation are resected. Definite internal fixation may be performed using lag screws or a buttress plate, from the small fragment set (The choice of implants depends on the size and bone quality of the PM fragment.). Buttress plate may prevent secondary displacement of the fragment, particularly in osteoporotic bone. Once the posterior malleolus fragment is secured, the fibular fracture is exposed through the sameincision, either medially or laterally to the peroneal tendons, following the fracture line. They are generally displaced medially to obtain good exposure of distal fractures and allow placement of lateral or posterolateral plates. Finally, the medial malleolus is addressed. The fixation of the medial malleolus can be 
difficult in the prone position but could be performed correctly, assistant holding the leg with the knee flexed and rotated internally or even patient can be placed in a supine position. The medial malleolus treated via standard medial approach and fixation can be done as per fracture pattern. The closure is performed in the usual manner [25] [34] [35] [36] [37] (Figures 1-6).

\section{Complication}

Complication of ankle fractures may be malunion, nonunion, infection, wound dehiscence, posttraumatic osteoarthritis, posttraumatic reflex dystrophy, deep venous thrombosis, embolism, compartment syndrome and nerve injury. A systemic review of 17 studies done by E. S. Veltman et al. which included 768 patients. Wound infection was present in 39 with deep infection for 7 cases and superficial infection for 32 cases were witnessed. Dehiscence was present among 16 patients. It required implant removal for 88 infected patients as a result of pain. A persisting intra-articular step-off of $>2 \mathrm{~mm}$ was noted in 32 patients. Non-union was present in 19 out of 768 patients. Posttraumatic osteoarthritis was present in 16 patients among all patients after a mean follow-up of 44

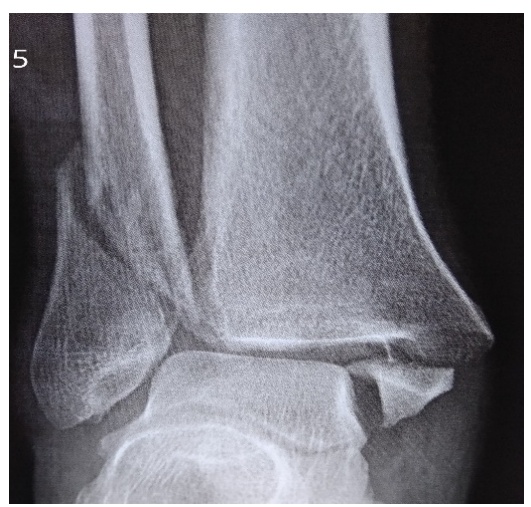

Figure 1. Anteroposterior radiograph of trimalleolus fracture (image taken from zhongda hospital).

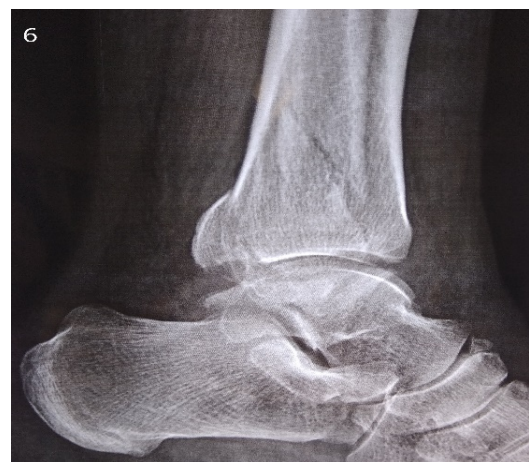

Figure 2. Lateral radiograph showing posterior malleolus fracture (image taken from zhongda hospital). 


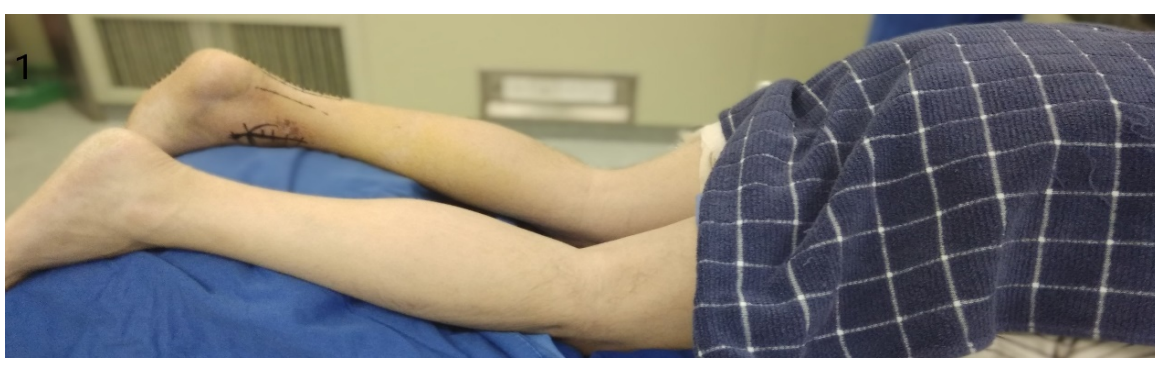

Figure 3. Patient with prone position with slightly knee flexed (image taken from zhongda hospital).

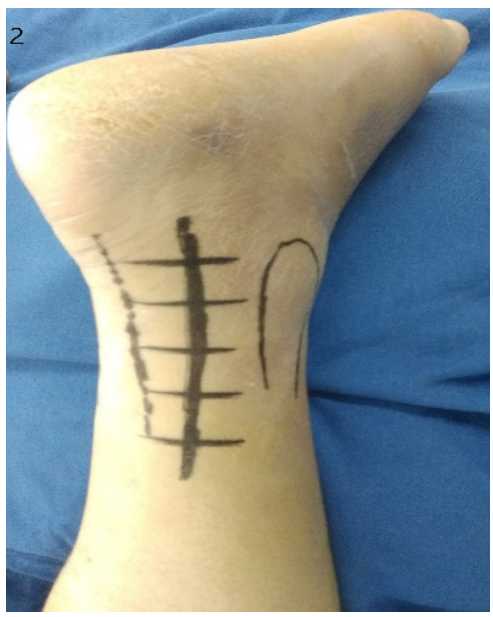

Figure 4. Outline of a longitudinal incision made between the Achilles tendon and posteriorborder of the fibula (image taken from zhongda hospital).

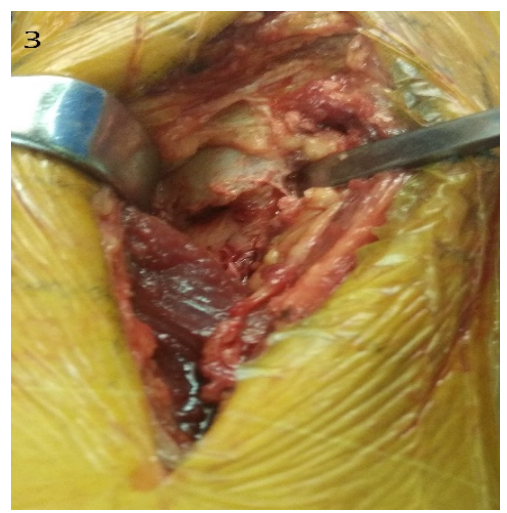

Figure 5. View of the posterior fragment in the interval between the peroneal tendons and the flexor hallucis longus (image taken from zhongda hospital).

months. Nine patients ( $1 \%$ of all patients) had complaints of posttraumatic reflex dystrophy. Persistent talar subluxation was present in seven patients (1\% of all patients). Two patients had persistent nerve damage [38]. 


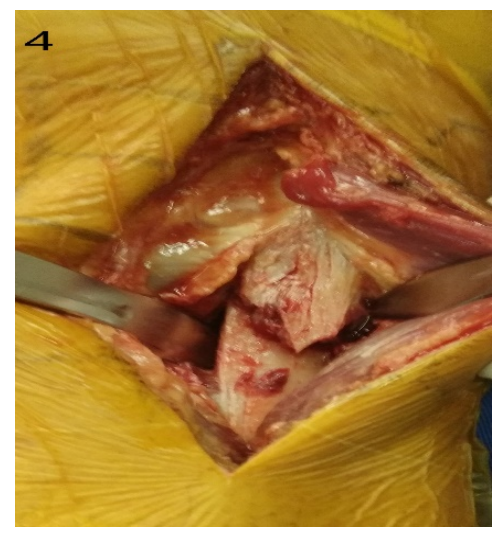

Figure 6. Exposure of fibula fracture through same incision (image taken from zhongda hospital).

\section{Posterolateral versus Other Approaches}

There are several studies describing surgical approaches to the posterior malleolus fracture. A long medial incision was used to reach the posterior fragment of the dislocated ankle. This method requires extensive soft tissue stripping of the fracture fragments [39]. Kao et al. described a posterior-medial-anterior approach to pilon fractures that uses a larger J-type incision that proximally starts posteriorly curving around the medial malleolus and distally over the dorsomedial foot [40]. A posteromedial incision is also indicated allowing fixation of the posterior and medial malleoli from the same incision [36] [40] [41]. Although, this approach has a limited visualization of the posterior malleolus fragment. Holt et al. had described an arthroscopically assisted reduction of the posterior malleolus [42]. Weber et al. described a case series of 9 patients who were treated with a combined posteromedial and posterolateral approaches [43]. Indirect reduction and screw fixation are minimally invasive in comparison to direct reduction through posterolateral approach. However, during anterior incision the removal of periosteal debris or blood clots are not satisfactory due to minimal visualization off ragments.

Previously, posterior malleolus surgical approaches required undue dissection or had inadequate visualization. Therefore, regarding the surgical method for posterolateral approach has several advantages. Importantly, it allows a direct inspection with proper reduction of the fragments. It is a well-known fact, articular surface is a basic principle in fracture reduction, and the posterolateral approach definitely supports the fact. This was exclusively presented in a study by Huber et al. [18]. They found that anatomical reduction of the posterior malleolus was more frequent with direct reduction ( $83 \%$ of cases) while compared with the standard indirect reduction and anteroposterior screws (27\% of cases). During delay in surgery, a direct cleaning and removal of callus and periosteum is possible. Finally, direct visualization allows for the joint to be inspected for osteochondral fragments, talar chondral damage or impaction injury. The fixed 
implants used for posterolateral approach is deep within the ankle which is important for good soft tissue coverage and also provides more comfort to patients.

\section{Discussion}

The treatment goal of an ankle fracture is the complete recovery with the purpose of the maximal possible function. Stable reduction, early weight bearing, and motion of the ankle joint are the most popular methods implemented. A proper reduction of ankle joint is very important because it is a small articular surface with a maximal heavy load bearer. Although there are studies confirming poor prognosis for the posterior malleolus fracture of the ankle, controversies still exist regarding the appropriate indication and its management. Indirect reduction of the fragment with an anterior approach and fixation with anteroposterior screws is one of the treatment modalities for PM fractures [34]. Due to possible periosteal interposition, hematoma, and callus plus in the delayed cases this method is not usually a success.

Previously, studies report no consensus on the optimal size of the fragment that should be fixed [44] hence with poor optimal indication and treatment algorithm. Traditionally, the size of the fragment was the main factor for operative indication. Many surgeons internally fix the posterior malleolus fracture if the fragments are larger than $25 \%-30 \%$ of the articular surface. However, a study by Gardner et al. [45] suggested, significant variations exist along with size considered for surgical fixation of posterior malleolus fractures. Harper and Hardin [46] found no significant statistical difference between the two treatment options while comparing the results of operative versus conservative treatment for PM fracture involving $25 \%$ or more of the articular surface. Heim et al. [47] recommends operative fixation for all Posterior malleolus fractures except for posterior rim fracture for tibia. Langenhujsen et al. [48] suggested reduction and fixation for all posterior malleolus fracture that remains displaced after ORIF of the lateral and medial malleolus.

With the advancement of the computed tomography scans on evaluating these fractures has revolutionized its use on orthopedic surgeries. Increasing application CT scans on an increasing number of clinical studies provide the clinicians a better understanding of factors that affect the treatment decision regardless of the singular size of the fragment entity to perform an operative treatment [49]. With surgeons becoming more familiar and minimal risk associated with the posterolateral approach has attracted investigators for implementing the posterolateral approach and fixation of the posterior malleolus from its posterior aspect. Miller et al. [50] followed up 31 patients who had undergone the posterolateral approach among which 9 had a posterior malleolus fracture, 14 had a syndesmotic injury without posterior malleolus fracture, and 8 had ankle dislocation with posterior malleolus fracture. All the patients underwent surgery and fixation with the posterolateral approach. It was observed, posterior malleolus stabilization of the syndesmotic injuries was equivalent to screw fixation. Fur- 
ther, recommended regardless of the size of the fracture fragment, an anatomic reconstruction should be performed by fixation using a posterolateral approach. In a study by Forberger et al. [51] which included 45 patients with the posterolateral surgical approach involving more than $25 \%$ of the articular surface or young patients with subluxation of the ankle more than $10 \%$ of the articular surface. The study states that the approach had a good exposure with stable fixation for the displaced posterior malleolus fragment and further with only a few complications.

Although several studies have suggested using the posterolateral approach for the treatment of posterior malleolus fractures, only a few have described the treatment outcomes. Abdelgawad et al. [36] reported on 12 cases of posterior malleolus fractures treated with a posterolateral approach. 10 patients were followed up, and none of 10 patients had developed delayed union, non-union, deep infection, or wound dehiscence. Mingo Robinet et al. [35] also treated 10 patients through the posterolateral approach and none of them had any postoperative complication. Benthien [52] only reported 3 cases among which all achieved satisfactory bone healing, anatomic alignment, and the full range of ankle motion. Erdem et al. [34] treated 40 patients through the posterolateral approach and reported that full union without any loss of reduction was obtained in 38 out of 40 patients. Recently, Q. Zhou et al. also treated 34 patients through the posterolateral approach and reported that no patient developed delayed union or nonunion [37].

\section{Conclusion}

In conclusion, the posterolateral approach provides an optimal exposure for the fixation of the posterior malleolus fracture. Further, this approach has an advantage of proper visualization of the fragments and better functional stability of the ankle joint. This technique also provides good coverage of soft tissue and stable fixation, with few postoperative complications.

\section{Acknowledgements}

We would like to thank the faculty members of Department of Orthopedic Surgery, Zhongda Hospital affiliated to Southeast University for their coordination. Further, we express our gratitude towards Shrestha Sachin Mulmi for his guidance.

\section{Conflicts of Interest}

The authors declare no conflicts of interest regarding the publication of this paper.

\section{References}

[1] Daly, P.J., Fitzgerald, R.H., Melton, L.J. and Llstrup, D.M. (1987) Epidemiology of Ankle Fractures in Rochester, Minnesota. Acta Orthopaedica Scandinavica, 58, 
539-544. https://doi.org/10.3109/17453678709146395

[2] Jensen, S.L., Andresen, B.K., Mencke, S. and Nielsen, P.T. (1998) Epidemiology of Ankle Fractures: A Prospective Population-Based Study of 212 Cases in Alborg, Denmark. Acta Orthopaedica Scandinavica, 69, 48-50. https://doi.org/10.3109/17453679809002356

[3] Thur, C.K., Edgren, G., Jansson, K.Å. and Wretenberg, P. (2012) Epidemiology of Adult Ankle Fractures in Sweden between 1987 and 2004: A Population-Based Study of 91,410 Swedish Inpatients. Acta Orthopaedica, 83, 276-281. https://doi.org/10.3109/17453674.2012.672091

[4] Court-Brown, C.M., McBirnie, J. and Wilson, G. (1998) Adult Ankle Fractures-An Increasing Problem? Acta Orthopaedica Scandinavica, 69, 43-47. https://doi.org/10.3109/17453679809002355

[5] Xu, H.L., Liu, L.M., Li, X., Zhang, D.Y., Fu, Z.G., Wang, T.B., et al. (2012) Multicenter Follow-Up Study of Ankle Fracture Surgery. Chinese Medical Journal, 125, 574-578.

[6] Chaput, H. (1907) Les fractures malléolaires du cou-de-pied et les accidents du travail. Masson, France.

[7] Cotton, F.J. (1915) A New Type of Ankle Fracture. Journal of the American Medical Association, 64, 318-321. https://doi.org/10.1001/jama.1915.02570300032010

[8] Adams, R. (1835) Ankle Joint, Abnormal Conditions. The Cyclopaedia of Anatomy and Physiology, 1, 1835-1836.

[9] Köken, M., Akşahin, E. and Çelebi, L. (2016) Posterior malleol kırıklarında güncel yaklaşımlar. Türk Ortopedi ve Travmatoloji Birliği Derneği, 15, 191-196.

[10] Gardner, M.J., Brodsky, A., Briggs, S.M., Nielson, J.H. and Lorich, D.G. (2006) Fixation of Posterior Malleolar Fractures Provides Greater Syndesmotic Stability. Clinical Orthopaedics and Related Research, 447, 165-171. https://doi.org/10.1097/01.blo.0000203489.21206.a9

[11] Hartford, J.M., Gorczyca, J.T., McNamara, J.L. and Mayor, M.B. (1995) Tibiotalar Contact Area. Contribution of Posterior Malleolus and Deltoid Ligament. Clinical Orthopaedics and Related Research, 320, 182-187.

[12] Macko, V.W., Matthews, L., Zwirkoski, P. and Goldstein, S. (1991) The Joint-Contact Area of the Ankle. The Contribution of the Posterior Malleolus. The Journal of Bone and Joint Surgery-American Volume, 73, 347-351. https://doi.org/10.2106/00004623-199173030-00005

[13] Hooff, V.D., Verhage, S.M. and Hoogendoorn, J.M. (2015) Influence of Fragment Size and Postoperative Joint Congruency on Long-Term Outcome of Posterior Malleolar Fractures. Foot and Ankle International, 36, 673-678. https://doi.org/10.1177/1071100715570895

[14] Xu, H.L., Li, X., Zhang, D.Y., Fu, Z.G., et al. (2012) A Retrospective Study of Posterior Malleolus Fractures. International Orthopaedics, 36, 1929-1936. https://doi.org/10.1177/1071100715570895

[15] Bartoníček, J., Rammelt, S. and Tuček, M. (2017) Posterior Malleolar Fractures: Changing Concepts and Recent Developments. Foot and Ankle Clinics, 22, 125-145. https://doi.org/10.1016/j.fcl.2016.09.009

[16] Grantham, S.A. (1990) Trimalleolar Ankle Fractures and Open Ankle Fractures. Instructional Course Lectures, 39,105-111.

[17] Jupiter, J.B., Levine, A.M., Trafton, P.G. and Browner, B.D. (2003) Skeletal Trauma: Basic Science, Management, and Reconstruction. WB Saunders, Philadelphia. 
[18] Huber, M., Stutz, P. and Gerber, C. (1996) Open Reduction and Internal Fixation of the Posterior Malleolus with a Posterior Antiglide Plate Using a Postero-Lateral Approach-A Preliminary Report. Foot and Ankle Surgery, 2, 95-103. https://doi.org/10.1046/j.1460-9584.1996.00012.x

[19] Tartaglione, J.P., Rosenbaum, A.J., Abousayed, M. and DiPreta, J.A. (2015) Classifications in Brief: Lauge-Hansen Classification of Ankle Fractures. Springer, Berlin.

[20] Goost, H., Wimmer, M.D., Barg, A., Kabir, K., Valderrabano, V. and Burger, C. (2014) Fractures of the Ankle Joint: Investigation and Treatment Options. Deutsches Arzteblatt International, 111, 377-388.

[21] Danis, D.R., Université de B., et al. (1949) Théorie et pratique de l'ostéosynthèse: par Robert Danis. Masson, Niort.

[22] Prasarn, M.L. and Lorich, D.G. (2017) Posterior Malleolus Fractures in Athletes. Operative Techniques in Sports Medicine, 25, 82-86. https://doi.org/10.1053/j.otsm.2017.03.007

[23] Lesic, A. and Bumbasirevic, M. (2004) Ankle Fractures. Current Orthopaedics, 18, 232-244. https://doi.org/10.1016/j.cuor.2004.03.001

[24] Haraguchi, N., Haruyama, H., Toga, H. and Kato, F. (2006) Pathoanatomy of Posterior Malleolar Fractures of the Ankle. The Journal of Bone and Joint Surgery, 88, 1085-1092. https://doi.org/10.2106/JBJS.E.00856

[25] Talbot, M., Steenblock, T.R. and Cole, P.A. (2005) Surgical Technique: Posterolateral Approach for Open Reduction and Internal Fixation of Trimalleolar Ankle Fractures. Canadian Journal of Surgery, 48, 487-490.

[26] Leveuf, J., Girode, C., Mornard, P., Monod, R. and Delbet, P. (1925) Traitement des fractures et luxations des membres. JAMA, 85, 1833.

[27] Lounsbury, B. and Metz, A. (1922) Lipping Fracture of Lower Articular End of Tibia. Archives of Surgery, 5, 678-690. https://doi.org/10.1001/archsurg.1922.01110150231010

[28] Ludloff, K. (1926) Zur Frage der Knöchelbrüche mit Heraussprengung eines hinteren Volkmann'schen Dreiecks. Zentralblatt für Chirurgie, 53, 390-391.

[29] Müller, M.E., Allgöwer, M., Schneider, R. and Willenegger, H. (1991) Manual der Osteosynthese. Springer, Berlin, Heidelberg, New York.

[30] Weber, M. and Ganz, R. (2003) Malunion Following Trimalleolar Fracture with Posterolateral Subluxation of the Talus-Reconstruction Including the Posterior Malleolus. Foot and Ankle International, 24, 338-344. https://doi.org/10.1177/107110070302400406

[31] Rammelt, S., Heim, D., Hofbauer, L., Grass, R. and Zwipp, H. (2011) Probleme und Kontroversen in der Behandlung von Sprunggelenkfrakturen. Der Unfallchirurg, 114, 847-860. https://doi.org/10.1007/s00113-011-1978-x

[32] Heim, U. (1982) Indikation und Technik der Stabilisierung des hinteren Kantendreiecks nach Volkmann bei Malleolarfrakturen. Hefte zur Unfallheilkunde, 85, 388-394.

[33] Nelson, M. and Jensen, N. (1940) The Treatment of Trimalleolar Fractures of the Ankle. Surgery, Gynecology and Obstetrics, 71, 509-514.

[34] Erdem, M.N., Erken, H.Y., Burc, H., Saka, G., Korkmaz, M.F. and Aydogan, M. (2014) Comparison of Lag Screw versus Buttress Plate Fixation of Posterior Malleolar Fractures. Foot and Ankle International, 35, 1022-1030. https://doi.org/10.1177/1071100714540893

[35] Mingo, R.J., Larrainzar, J.A. and Cruz, J.V. (2012) Posterolateral Approach in Tri- 
malleolar Ankle Fractures. Surgical Technique. Revista Española de Cirugía Ortopédica y Traumatología (English Edition), 56, 313-318. https://doi.org/10.1016/j.recote.2012.05.008

[36] Abdelgawad, A.A., Kadous, A. and Kanlic, E. (2011) Posterolateral Approach for Treatment of Posterior Malleolus Fracture of the Ankle. The Journal of Foot and Ankle Surgery, 50, 607-611. https://doi.org/10.1053/j.jfas.2011.04.022

[37] Zhou, Q., Lu, H., Wang, Z., Yu, S. and Zhang, H. (2017) Posterolateral Approach with Buttress Plates and Cannulated Screw Fixation for Large Posterior Malleolus Fractures. The Journal of Foot and Ankle Surgery, 56, 1173-1179. https://doi.org/10.1053/j.jfas.2017.05.028

[38] Veltman, E.S., Halma, J.J. and de Gast, A. (2016) Longterm Outcome of 886 Posterior Malleolar Fractures: A Systematic Review of the Literature. Foot and Ankle Surgery, 22, 73-77. https://doi.org/10.1016/j.fas.2015.05.003

[39] Shelton, M. (1983) Open Reduction and Internal Fixation of Comminuted Trimalleolar Fracture of the Ankle. Strategies Orthop Surg, 2, 3.

[40] Kao, K.F., Huang, P.J., Chen, Y.W., Cheng, Y.M., Lin, S.Y. and Ko, S.H. (2000) Postero-Medio-Anterior Approach of the Ankle for the Pilon Fracture. Injury, 31, 71-74. https://doi.org/10.1016/S0020-1383(99)00202-8

[41] Amorosa, L.F., Brown, G.D. and Greisberg, J. (2010) A Surgical Approach to Posterior Pilon Fractures. Journal of Orthopaedic Trauma, 24,188-193. https://doi.org/10.1097/BOT.0b013e3181b91927

[42] Holt, E.S. (1994) Arthroscopic Visualization of the Tibial Plafond during Posterior Malleolar Fracture Fixation. Foot and Ankle International, 15, 206-208. https://doi.org/10.1177/107110079401500409

[43] Weber, M. (2004) Trimalleolar Fractures with Impaction of the Posteromedial Tibial Plafond: Implications for Talar Stability. Foot and Ankle International, 25, 716-727. https://doi.org/10.1177/107110070402501005

[44] Bekerom, M.P., Haverkamp, D. and Kloen, P. (2009) Biomechanical and Clinical Evaluation of Posterior Malleolar Fractures. A Systematic Review of the Literature. Journal of Trauma and Acute Care Surgery, 66, 279-284. https://doi.org/10.1097/TA.0b013e318187eb16

[45] Gardner, M.J., Streubel, P.N., McCormick, J.J., Klein, S.E., Johnson, J.E. and Ricci, W.M. (2011) Surgeon Practices Regarding Operative Treatment of Posterior Malleolus Fractures. Foot and Ankle International, 32, 385-393.

https://doi.org/10.3113/FAI.2011.0385

[46] Harper, M.C. and Hardin, G. (1988) Posterior Malleolar Fractures of the Ankle Associated with External Rotation-Abduction Injuries. Results with and without Internal Fixation. The Journal of Bone and Joint Surgery, 70, 1348-1356. https://doi.org/10.2106/00004623-198870090-00012

[47] Heim, U.F. (1989) Trimalleolar Fractures: Late Results after Fixation of the Posterior Fragment. Orthopedics, 12, 1053-1059.

[48] Langenhuijsen, J.F., Heetveld, M.J., Ultee, J.M., Steller, E.P. and Butzelaar, R.M. (2002) Results of Ankle Fractures with Involvement of the Posterior Tibial Margin. Journal of Trauma and Acute Care Surgery, 53, 55-60. https://doi.org/10.1097/00005373-200207000-00012

[49] Ferries, J.S., DeCoster, T.A., Firoozbakhsh, K.K., Garcia, J.F. and Miller, R.A. (1994) Plain Radiographic Interpretation in Trimalleolar Ankle Fractures Poorly Assesses Posterior Fragment Size. Journal of Orthopaedic Trauma, 8, 328-331.

https://doi.org/10.1097/00005131-199408000-00009 
[50] Miller, A.N., Carroll, E.A., Parker, R.J., Helfet, D.L. and Lorich, D.G. (2010) Posterior Malleolar Stabilization of Syndesmotic Injuries Is Equivalent to Screw Fixation. Clinical Orthopaedics and Related Research, 468, 1129-1135. https://doi.org/10.1007/s11999-009-1111-4

[51] Forberger, J., Sabandal, P.V., Dietrich, M., Gralla, J., Lattmann, T. and Platz, A. (2009) Posterolateral Approach to the Displaced Posterior Malleolus: Functional Outcome and Local Morbidity. Foot and Ankle International, 30, 309-314. https://doi.org/10.3113/FAI.2009.0309

[52] Benthien, R.A. (2014) The Posterolateral Approach to the Posterior Malleolus: An Alternative Surgical Strategy for Unstable Trimalleolar Ankle Fractures. Techniques in Orthopaedics, 29, 8-12. https://doi.org/10.1097/BTO.0000000000000062

\section{Abbreviation}

PM: posterior malleolus

PITFL: posterior inferior tibiofibular ligament

TFS: tibiofibular syndesmosis

AITFL: anterior inferior tibiofibular ligament

CT: computed tomography

MRI: magnetic resonance imaging 